\title{
Theoretical study of short- and long-range forces and atom transfer in scanning force microscopy
}

\author{
S. Ciraci* and E. Tekman* \\ Department of Physics, Bilkent University, Bilkent 06533, Ankara, Turkey \\ and IBM Research Division, Zurich Research Laboratory, 8803 Rüschlikon, Switzerland
}

\author{
A. Baratoff \\ IBM Research Division, Zurich Research Laboratory, 8803 Rüschlikon, Switzerland
}

Inder P. Batra

IBM Research Division, Almaden Research Center, 650 Harry Road, San Jose, California 95120-6099

(Received 30 December 1991)

\begin{abstract}
We investigate the interaction energy, the short-range force components, and the electron potential between two $\mathrm{Al}(001)$ slabs, which mimic a blunt tip close to an atomically corrugated sample in scanning force microscopy. The adhesive energy and perpendicular force calculated using the selfconsistent-field pseudopotential method in the local-density approximation are site dependent, but can be accurately represented by a universal function in terms of scaled variables in the attractive range. The lateral force which determines friction variations on an atomic scale is not simply proportional to the perpendicular force and is typically one order of magnitude smaller. At larger separations the effect of the total long-range Van der Waals force and of its gradient are estimated to be small for a sharp conical support tip, but quite appreciable for a rounded support tip with a radius as small as $200 \AA$. By calculating the interaction energy of an $\mathrm{Al}$ atom between two slabs, we also study the possibility of single-atom transfer between tip and sample, and show that the double well in the interaction energy collapses into a single minimum at a slab separation larger than two bulk interlayer spacings. The atom is preferentially located on the side of the deeper minimum, but can hop between the two wells at finite temperatures. Moreover, the position of the deeper minimum relative to the electrodes can vary as the tip is scanned against the sample. Finally we explore possible relations between the short-range perpendicular force and the tunneling conductance through the potential barrier between two semi-infinite jellium slabs as a function of their separation.
\end{abstract}

\section{INTRODUCTION}

The interaction energy and the force derived thereof are important effects of the mutual influence of tip and sample in scanning force microscopy (SFM) (Refs. 1 and 2) and even scanning tunneling microscopy (STM) (Refs. 3-5) when these properties show significant variations with tip position. The sign and the character of the force vary according to the value of the tip-sample separation $z$. At large $z$ (excluding charging effects) it is Van der Waals attraction (VdW) in origin, and can generally be attributed to correlations between electronic fluctuations in the electrodes. When referred to individual atoms, it is weak and practically uncorrugated, but owing to its long-range (inverse-power-law) character it may be large for the whole tip. At small separations of a few interatomic distances short-range forces of quantum-chemical origin eventually dominate the $\mathrm{VdW}$ force. The former force can be calculated with reasonable accuracy in the local-density approximation (LDA) and can be explicitly divided into electron-mediated attraction and ion-ion repulsion. Since wave functions decay exponentially in the intervening potential barrier, this force is short ranged.
In the case of metals, to which we restrict the discussion, the perpendicular component $F_{s \perp}(z)$ becomes increasingly attractive with decreasing separation, passes through a minimum, then decreases and becomes increasingly repulsive. At the crossover from attraction to repulsion $z=z_{e}$, the interaction energy $E_{i}\left(z=z_{e}\right)$ (excluding VdW interaction) of an atomically sharp tip has a minimum corresponding to a binding energy in the range of $1 \mathrm{eV} /$ atom typical of a chemical bond. The maximum attractive force is $\sim 1 \mathrm{nN} /$ atom for typical metal-sample and metal-tip systems. Weaker but nevertheless significant lateral forces arise when the tip is positioned off high-symmetry positions. These lateral forces are fundamentally conservative, but, in combination with deformations (even purely elastic), can produce instabilities, hysteresis, and losses via energy transfer to shear modes, resulting in an average friction force of nonconservative nature.

The effects of tip-sample interaction in SFM and in STM have been attracting a growing interest. ${ }^{6-8}$ A quantitative treatment of tip-sample interaction for a given tip structure requires detailed calculations. Even though the detailed atomic structure of a tip is usually unknown, is 
affected by its sharpening process, and can change during measurements, we can theoretically analyze specific arrangements to reveal fundamental aspects, and to clarify some of the outstanding issues in SFM and STM. The particular issues we are addressing are (i) the distance dependence and relative importance of short- and long-range forces, (ii) the same aspects in reference to lateral forces, (iii) the relative importance of different contributions and of individual atoms or layers, (iv) the stability of the outermost tip atom and the energy barrier for its transfer between tip and sample, and (v) the relation between the short-range perpendicular force and the conductance. Our results for the interaction energy, short-range force, and electron potential are obtained from self-consistent-field (SCF) calculations with nonlocal ionic pseudopotentials performed within the LDA. ${ }^{9}$ The sample is represented as a rigid slab of five $\mathrm{Al}(001)$ layers; the tip is assumed to be blunt (flat) and is also represented by a rigid $\mathrm{Al}(001)$ slab with a variable number of layers. By assuming that both tip and sample consist of the same kind of atomic layers, we are able to use periodic boundary conditions and to express the wave functions of the combined system in terms of a plane-wave basis. For comparison we computed the Van der Waals force for macroscopic support tips of different shapes in a continuum approximation. Finally we carried out force, transmission, and conductance calculations between two parallel semi-infinite electrodes by using a variational jellium approximation. Preliminary results have recently been reported. ${ }^{10}$

\section{SHORT-RANGE INTERACTION ENERGY AND PERPENDICULAR FORCE}

The interaction energy $E_{i}(z)$ (we define $z$ as the distance between the outermost layers) between the slabs representing sample and tip is extracted from totalenergy calculations ${ }^{11}$ with a kinetic-energy cutoff $\mid \mathbf{k}+$ $\left.\mathbf{G}\right|^{2} \leq 8$ Ry which was checked to provide reasonable results for bulk Al. Figure 1 illustrates the dependence of $E_{i}(z)$ calculated for a four-layer tip slab with the toplayer atom facing a hollow $(H)$ or top $(T)$ site of the sample. The variation of $E_{i}(z)$ and its minimum value ${ }^{12}$ minus the adhesion energy of the slabs $E_{b}=-E_{i}\left(z=z_{e}\right)$ $[1.37 \mathrm{eV} /$ cell (Refs. 11 and 12) for the $H$ site and 0.92 $\mathrm{eV} /$ cell for the $T$ site], exhibit a significant site dependence at small separation even for the simple metal surfaces considered here. Compared to our earlier results for $\mathrm{Al}(111),{ }^{8}$ the difference between the $H$ and $T$ geometries, as expected, becomes larger in the case of a more corrugated $\mathrm{Al}(001)$ surface. At the $H$ site, $E_{b}$ is larger since the resulting stacking corresponds to the natural one of $\mathrm{Al}(001)$ layers in bulk $\mathrm{Al}$. Moreover, maximum adhesion occurs close to $z=d_{0}=3.8$ a.u., the interlayer spacing. The apparent minimum of $E_{i}(z)$ at the $T$ site occurs at a separation somewhat smaller than the nearest-neighbor distance of bulk Al. It is actually a saddle point of the full three-dimensional energy surface, and hence corresponds to an unstable situation. In other words, $E_{i}(z)$ becomes lower for small lateral displacements. The binding energies calculated here correspond to rigid slabs, and would

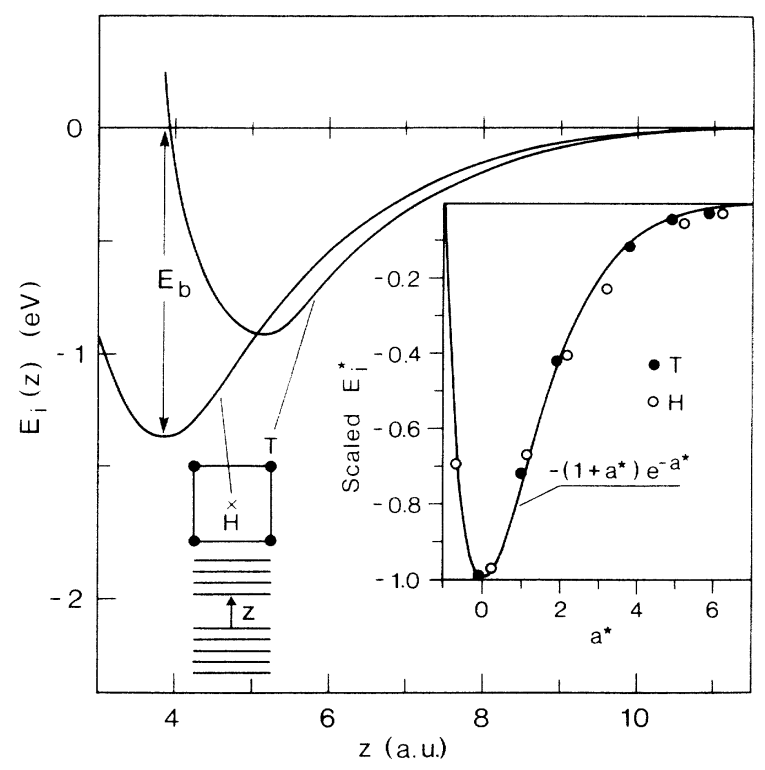

FIG. 1. Interaction energy $E_{i}$ versus separation $z$ between two rigid $\mathrm{Al}(001)$ slabs for the outermost atom of the tip slab facing the hollow $(H)$ or the top $(T)$ sites. The $z$ axis is perpendicular to the (001) plane. $E_{b}$ is the binding energy per unit cell. Top and side views of the geometric arrangement appear below. Inset. Scaled energy $E_{i}^{*}$ versus scaled separation $a^{*}$ (symbols) compared to the universal binding relation (full curve) proposed by Rose et al. (Ref. 14).

be further lowered if the atomic positions or interlayer distances were allowed to relax. $E_{b}$ shows only small variations with the number of layers in the tip slab. For example, for the $T$ site $E_{b}$ increases by $0.15 \mathrm{eV} /$ cell in going from a monolayer to a two-layer slab, but decreases by $0.05 \mathrm{eV} /$ cell in going from a two-layer slab to a fourlayer slab. For the $H$ site these variations are 0.11 and $0.10 \mathrm{eV} /$ cell, respectively. In the case of Morse-like pairwise interatomic potentials, $E_{b}$ is expected to decrease slightly with increasing number of layers and then to saturate. The nonmonotic dependence of $E_{i}$ is reminiscent of the oscillations of the work function and surface energy calculated earlier for thin $\mathrm{Al}(111)$ slabs. ${ }^{13}$ These oscillations, characteristic of metallic slabs, are interpreted as the manifestation of quantum size effects due to subbands successively dipping below the Fermi level as the slab thickness is increased. Such discrete subbands arise from confinement in the $z$ direction within each slab. In the present case such oscillations affect the potential barrier, and hence the decay constant of the wave function of the highest occupied subband, and in turn the interaction energy $E_{i}(z)$.

As seen in Fig. 1, the dependence of $E_{i}(z)$ calculated within the LDA is strong but short ranged, essentially because the total energy of the tip-sample system for a given separation is determined by charge-density overlap with only small deviations due to adhesive bond formation. It is therefore reasonable to expect that for $z>z_{e}, E_{i}(z)$ can be approximated by an exponential function. As a matter of fact, Rose et al. ${ }^{14}$ offered evi- 
dence and an approximate justification for a simple universal scaling relation in terms of the Rydberg function, $E_{i}^{*}=-\left(1+a^{*}\right) \exp \left(-a^{*}\right)$, for the interaction energy versus distance dependence of metallic and even covalently bound systems, including bulk crystals, parallel surfaces, adatoms, and diatomic molecules. The energy and distance are expressed in terms of the dimensionless variables $E_{i}^{*}=E_{i} / E_{b}$ and $a^{*}=\left(z-z_{e}\right) / \lambda$, respectively, where $\lambda$ is either taken proportional to the Thomas-Fermi screening length $\lambda_{\text {TF }}$ or treated as a fitting parameter related to the curvature of $E_{i}(z)$ near its minimum. It is remarkable that such a simple relation provides good fits for a large variety of systems and constituents even well away from $z=z_{e}$. For $\mathrm{Al}(001)$ slabs we find that our scaled energies also. fit well to the Rydberg function with $\lambda \simeq 1$ a.u. for both $H$ and $T$ sites, as seen in the inset to Fig. 1, especially in the range $z>z_{e}$ where the gradient of the attractive perpendicular force is positive. The systematic deviation apparent for the $H$ site indicates that a slightly longer $\lambda$ would be more appropriate in that case.

The preceding value of $\lambda$ should be compared to $\lambda_{\mathrm{TF}}=$ 0.91 a.u., calculated from the average electron density of bulk $\mathrm{Al}$, and to the values $\lambda_{b}=0.64$ a.u. and $\lambda_{s}=1.25$ a.u. proposed by Rose et al. to describe bulk cohesion and adhesion between the most densely packed (111) surfaces of $\mathrm{Al}$, respectively. ${ }^{14}$ Although these authors claim that $\lambda_{b} / \lambda_{s} \simeq 0.48 \pm 0.05$ for 28 different metals, their estimates of $\lambda_{s}$ are subject to a large uncertainty because they are based on surface energies obtained from experiments on polycrystals representing unknown averages over different low-index crystal faces. We therefore prefer to avoid the pitfall of misinterpreting the discrepancy between $\lambda_{s}$ and our fitted value for $\mathrm{Al}(001)$.

Later, the same researchers extended their treatment to the energetics of crystals perturbed by defects and applied this "equivalent-crystal theory" (ECT) to compute surface energies of different metals starting from bulk properties alone. ${ }^{15}$ For $\mathrm{Al}(001)$, in particular, they found a value corresponding to an adhesion energy of 0.65 $\mathrm{eV} /$ surface atom (for two surfaces ${ }^{12} 1.3 \mathrm{eV} /$ cell), which is close to ours for the $H$ site and also to the experimental one (subject to the above-mentioned uncertainties) of 0.6 $\mathrm{eV} /$ surface atom. Quite recently the same researchers applied their ECT to compute the interaction energy $E_{i}(z)$ versus separation between identical close-packed surfaces of different metals, as well as a metal adatom on one of the surfaces facing the other one. In these two situations representing the extremes of a blunt and sharp tip in SFM, Banerjea, Smith, and Ferrante ${ }^{16}$ found that $E_{i}(z)$ could be accurately fitted by the Rydberg function, even with the adatom facing sites of different symmetry. Unfortunately, they neither compared different sites on the same surface, nor considered $\mathrm{Al}(001)$, so that our finding of a nearly common value of $\lambda$ for different sites on this surface and its value remain to be checked against ECT. While it may be premature to draw general conclusions on the basis of the few available comparisons of the SCF calculations of $E_{i}(z)$ with the Rydberg function, ${ }^{10,17}$ our results reinforce the conclusion ${ }^{16}$ that this universal dependence provides a useful fit to the interaction energy versus position of the outermost tip atom(s) in SFM.
Moreover, ECT offers a promising avenue towards the estimation of the parameters $E_{b}, z_{e}$, and $\lambda$ and their site dependence.

The short-range force on an individual atom $j$ of the tip can be calculated either from the derivative of the calculated interaction energy, i.e., $\mathbf{F}_{s j}(\mathbf{r})=-\nabla_{j} E_{i}(\mathbf{r})$, or more conveniently from $-\left\langle\nabla_{j} H_{\mathrm{LDA}}\right\rangle .{ }^{18}$ Indeed, once self-consistency has been achieved, changes in the wave function due to displacement of nuclei do not contribute to the force, since the eigenfunctions are obtained variationally. As a consequence the force $\mathbf{F}_{\boldsymbol{s} j}$ can be expressed as the sum of the electron-mediated attraction (in which the electron density is calculated from the self-consistent wave functions),

$$
\int\left[\rho_{s}(\mathbf{r})+\Delta \rho(\mathbf{r})\right]\left\{\frac{\partial}{\partial \mathbf{R}_{j}}\left[\frac{Z_{j}}{\left|\mathbf{R}_{j}-\mathbf{r}\right|}\right]\right\} d \mathbf{r}
$$

and the ion-ion repulsion

$$
-\sum_{s} \frac{\partial}{\partial \mathbf{R}_{j}}\left\{\frac{Z_{j} Z_{s}}{\left|\mathbf{R}_{j}-\mathbf{R}_{s}\right|}\right\}
$$

which compensate each other almost completely at large separation. In the above equations, $\mathbf{R}_{s}\left(\mathbf{R}_{j}\right)$ is the position vector and $Z_{s}\left(Z_{j}\right)$ is the core charge of a sample (tip) ion, $\rho_{s}(\mathbf{r})$ is the valence charge density of the bare sample alone, and $\Delta \rho(\mathbf{r})$ denotes the change in charge density due to the tip-sample interaction. ${ }^{7}$

In Fig. 2 we illustrate the variation of the perpendicular and parallel components of the total short-range force on one atom of a single-layer tip slab for different lateral positions relative to the sample slab. The strongest attraction occurs at the smallest $z=z_{m}$ at the $H$ site. As the tip atom is shifted from the $H$ towards the $T$ site, the minimum and the zero crossing $\left(z=z_{e}\right)$ of the perpendicular force gradually shifts to larger $z$ and concomitantly the strength of the attraction decreases. The calculated curves indicate a corrugation $\Delta z=1.2$

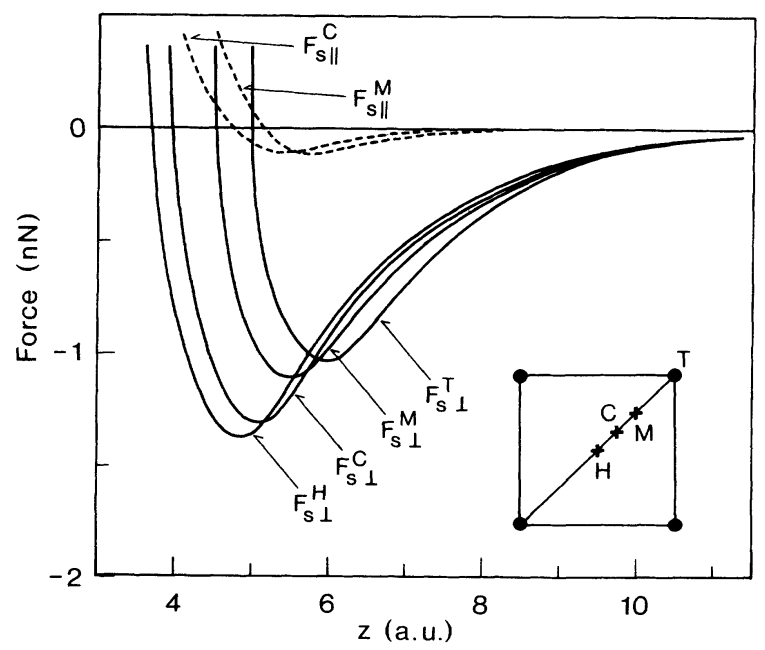

FIG. 2. Perpendicular $F_{s \perp}$ and lateral $F_{s \|}$ forces (in $\mathrm{nN} /$ atom) on the single-layer "tip slab" versus separation $z$, calculated for the tip atom facing $H, C, M$, and $T$ sites shown in the inset. 
a.u. at a constant loading force $F_{s \perp}$ in the range of \pm 1 $\mathrm{nN} /$ atom. The different $F_{s \perp}$ curves merge above $z \simeq 10$ a.u., slightly beyond the separation at which a real potential barrier exceeding the Fermi energy $E_{F}$ appears between the slabs. These features are analogous to those found and discussed in our earlier work on tip-sample interaction, ${ }^{7,8,10}$ whereas the crossing(s) beyond $z_{m}$ are system specific.

\section{SHORT-RANGE LATERAL FORCE}

Measurements of the lateral forces acting on the tip in SFM as a function of perpendicular loading force and scan velocity have revealed atomic-scale variations on graphite. ${ }^{19}$ Zhong and Tomanek ${ }^{20}$ have provided a theoretical estimate of the average friction coefficient $\mu$ from $E_{i}(z)$ calculated for a commensurate Pt monolayer sliding against graphite in the repulsive range. They assumed that in the limit of slow tracking velocity the energy increase in going from the $H$ to the $T$ site is stored conservatively in bending the cantilever and is then fully dissipated in the opposite sequence. This is a rather unrealistic assumption, however. Indeed, experimental data show an average nonconservative hysteretic component superposed on a conservative component, modulated with the lateral periodicity of the sample surface. An important factor pointed out by Mate et al. ${ }^{19}$ is that the strain stored in the vicinity of the tip may only be partially released in the observed stick-slip motion. A full account of the friction arising in the course of the observed stick-slip motion should include the dissipation of energy by phonons and other excitations in both tip and sample, ${ }^{21}$ and requires further work. In the present study we only calculate the conservative lateral forces $F_{s \|}(z)$, as illustrated in Fig. 2 (dashed curves) for the tip atom facing the $M$ and $C$ sites indicated by the inset. By symmetry they are directed along the diagonal $H T$ and vanish if the tip atom is facing the $H$ and $T$ sites of the sample. They are one order of magnitude smaller than the perpendicular forces in a wide range of $z$. This is likely to be a generic feature which can qualitatively be understood as follows. In an effective pair-interaction picture, which may be approximately justified by neglecting $\Delta \rho$ and representing $\rho_{s}$ in Eq. (1) by a superposition of spherical atomiclike densities, the vector contributions from all neighboring sample atoms tend to add up in $\mathbf{F}_{s \perp}$, but tend to cancel out in $\mathbf{F}_{s \|}$. Furthermore, $F_{s \|}(z)$ is not proportional to $F_{s \perp}(z)$, although it exhibits a similar overall dependence and changes sign at a value $z=z_{\|}$ between $z_{e}$ and $z_{m}$. For $z \geq z_{\|}, F_{s \|}$ is directed towards $T$, but for $z<z_{\|}$it is reversed, consistent with the bulk stacking of $\mathrm{Al}$ layers in the (001) direction for $z=z_{e}$. $F_{s \|}$ is finite at $z=z_{m}$ (where $F_{s \perp}$ has the strongest attraction) even though $F_{s \perp}=0$. These features can be understood in terms of the three-dimensional interaction energy $E_{i}(\mathbf{r})$, the minimum of which at fixed $z$ switches from $T$ to $H$ as $z$ is increased (see Fig. 1).

\section{LONG-RANGE FORCE}

Tunneling and short-range interactions are usually dominated by poorly controlled and characterized pro- tuberances consisting of a few atoms on much larger "support" tip. The importance of the VdW interaction in SFM was recognized earlier, and it was argued that depending on the overall shape of the support tip, the atom at the apex of the tip can experience strong repulsion even leading to irreversible deformations while atoms further away from the apex experience an overall attraction. ${ }^{7}$ Building up on previous work concerned with $\mathrm{VdW}$ interaction between macroscopic bodies, various authors ${ }^{17,22-24}$ have recently investigated the importance of that interaction in SFM. Existing treatments rely on summing the asymptotic interaction law $-C_{n} r^{-n}$ over the volume of the tip (and of the sample in the first case mentioned below). This interaction law describes the VdW interaction energy at a distance $r$ between two atoms $(n=6), 22$ between an atom and a polarizable flat surface $(n=3),{ }^{24}$ or between two such surfaces $(n=2) .{ }^{23}$ In the last two cases the quoted laws are presumed to hold at distances sufficiently large that details of atomic structure cease to matter and a continuum description based on integration becomes justified for bodies with cross section varying slowly on that scale. ${ }^{25,26}$ Although the $z$ dependence of the net interaction resulting from these various approaches is the same for electrodes of a given shape, the calculated strengths $C_{n}$ depend on the tip and sample materials and can differ significantly. This is so because, for instance, the polarizability of a metallic tip or sample is larger than the sum of the atomic polarizabilities of its free constituents. Thus it is by no means clear whether the prescription proposed in Ref. 23 is adequate for metal tips. Indeed, for metal electrodes, a generalized Lifshitz approach, ${ }^{27-29}$ appears to be most appropriate. It is expected to be valid at separations such that wave-function overlap and, hence, exchange effects become negligible, whereas remaining electron correlation effects require a nonlocal description. At separations $z \gtrsim c / \omega_{p} \sim 10^{3}$ a.u. the inverse power of the interaction law is increased by unity, owing to electromagnetic retardation, ${ }^{24}$ but such effects are of academic interest in the context of SFM with atomic or nanometer lateral resolution, which requires much smaller $z$. In that range, this theory can be somewhat improved by taking spatial dispersion into account, for instance, by including the next term in an inverse-power-law expansion or absorbing it into a shift $z_{j} \rightarrow z_{j}-z_{0} \cdot{ }^{28,29}$ [Note that the separation $z$ defined at the outset is between the outermost atomic layers, but in theoretical treatments based on the jellium model it is more appropriate to consider the distance $z_{j}$ between jellium edges; in the case of $\mathrm{Al}(001), z_{j} \simeq z-d_{0}$ in a.u.] Adapting the results of these treatments to our situation, we conclude that $z_{0}$ must be smaller than twice the separation of the static image plane from the jellium edge of one surface, i.e., at most $\simeq 3$ a.u. in the case of $\mathrm{Al}$ (jellium density parameter $r_{s} \simeq 2$ ). ${ }^{29}$ More ambitious attempts to bridge the gap between the resulting Lifshitzlike asymptotic expression and short distances at which the LDA is believed to become adequate, which were mostly pursued in the context of He-atom interactions with metal surfaces, ${ }^{30}$ are to our knowledge still frought with uncertainties. They all replace the apparent divergence as $z_{j} \rightarrow z_{0}$ by a smooth crossover. The more ad hoc 
approach which we prefer is to imagine a smooth interpolation between the shifted Lifshitz expression and our LDA results, as first attempted by Inglesfield. ${ }^{27}$ Using his results as a guide, we note that the VdW interaction can to a good approximation be expressed in terms of the change in zero-point energy of the surface-plasmon modes of the electrodes, which are coupled through their electric fields. ${ }^{27}$ In the case of flat parallel electrodes, the VdW interaction is dominated by the contribution from longwavelength modes at separations $z_{j} \gtrsim 10$ a.u.; in this limit the Lifshitz expression is recovered. However, the infinite-barrier model for the surface response on which Inglesfield's work is based has been recognized as inadequate. In particular, it leads to an unphysical negative shift $z_{0}$, which has been corrected in later work. ${ }^{28-30}$

In order to simplify matters, we henceforth present results computed from the Lifshitz expression ${ }^{25}$ with the provision that they present overestimates for $z_{j} \lesssim 10$ a.u. and that the origin of $z_{j}$ is likely to be decreased by an additional $z_{0}<3$ a.u. with respect to $z-d_{0}$. Such shifts can have important consequences for $z_{j}$ values of the same order. On the other hand, the origin may be effectively shifted to a larger value of $z-d_{0}$, depending on the extent of the atomic-scale protuberance, which controls short-range interactions. The shape of the tip has a crucial influence on the magnitude and $z$ dependence of the total interaction. In SFM experiments, not only the VdW force but also its gradient can be important. Even at large $z$, where the short-range forces are negligible, the gradient of the $\mathrm{VdW}$ force can become relevant if it exceeds the spring constant of the cantilever. This causes a mechanical instability in which the tip jumps to the range $z \simeq z_{e}$. At such small $z$, the gradient of the short-range force can dominate that due to the $\mathrm{VdW}$ force. ${ }^{17,22,24}$

In what follows, we confirm that apart from such instabilities, which can be avoided by using a stiff cantilever, the VdW interaction is unlikely to dominate the force and force gradient in measurements with sharp tips in SFM. ${ }^{17}$ To this end we consider semi-infinite tips with conical and hemispherical ends and a cylindrical shank, as illustrated in Fig. 3. By varying the cone angle $\alpha$, but keeping the diameter fixed for the former, and by varying the diameter for the latter, we are able to cover a wide range of tip geometries. Since the system consists of two electrodes, one of which is flat on the relevant scale (the sample surface), it is most appropriate to integrate the Lifshitz formula for an atom interacting with a polarizable surface and over the tip volume to obtain the total VdW interaction energy ${ }^{26}$

$$
E_{W}=-\frac{A}{6 \pi} \int_{V_{\mathrm{tip}}} \frac{d \mathbf{r}}{[z(\mathbf{r})]^{3}}
$$

where $z(\mathbf{r})$ is the height of the differential volume element located at $\mathbf{r}$ in the tip, and $A=0.36, a J=2.25 \mathrm{eV}$ is the Hamaker constant, as found from Ref. 27. There are different definitions of the Hamaker constant in literature, and we choose Israelachvili's definition, ${ }^{26}$ which is also adopted by Goodman and Garcia ${ }^{23}$ and Hartmann. ${ }^{24}$ The Hamaker constants estimated in the former work are consistently higher for reasons that are not altogether clear. In the present study we actually integrated the Lifshitz expression perpendicular to $z$, but performed a summation over a sequence of disks of thickness equal to the interlayer spacing of $\mathrm{Al}(001)$ to partially represent the effect of atomic structure. The radii of the disks are determined by the geometrical tip shapes described above. Because values of $z(\mathbf{r})$ giving the main contribution to Eq. (3) scale with the minimum separation $z_{j},{ }^{24}$ the difference between summation and integration becomes small for large $z_{j} \gtrsim 10$ a.u., where the asymptotic expression is accurate. As emphasized above, for small separations our results overestimate the VdW interac-
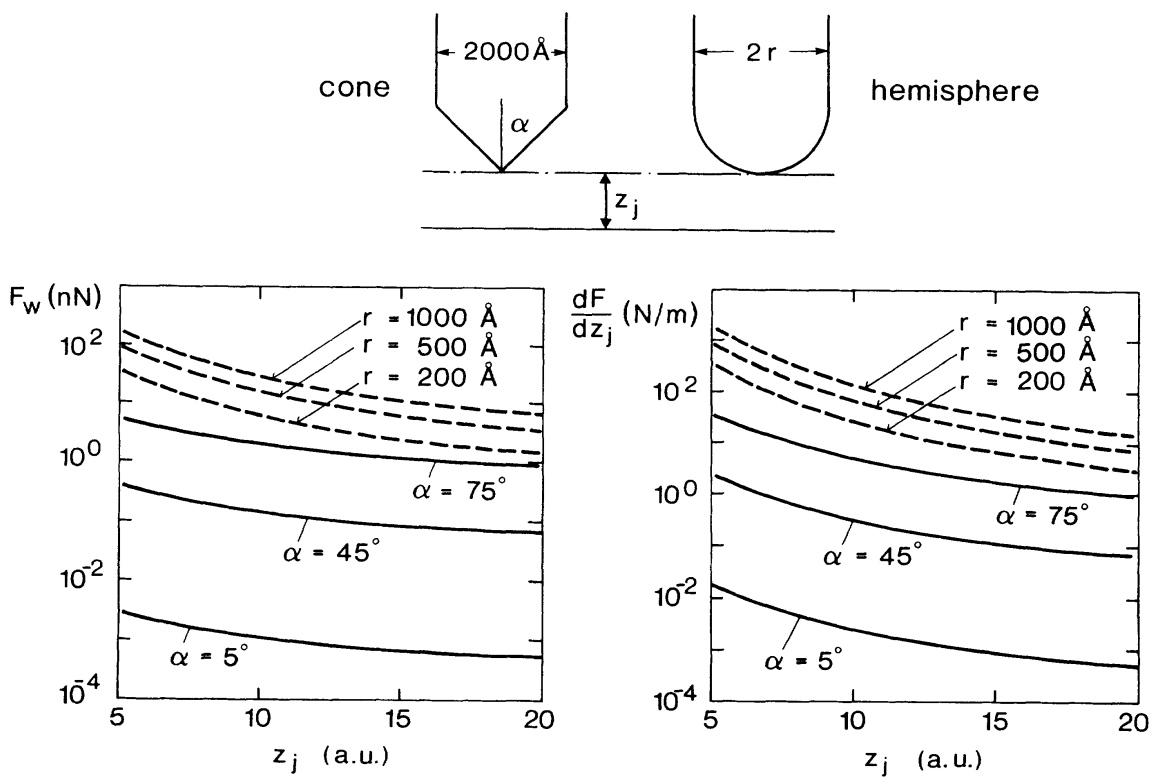

FIG. 3. Van der Waals force $F_{W}$ and its gradient $d F_{W} / d z_{j}$ calculated for different tip geometries described in the top panel. The tip sample separation $z_{j}$ is measured from the jellium edges of both electrodes. The Hamaker constant used is $3.6 \times 10^{-19}$ J. 
tion for a given $z_{j}$ and the difference $z_{j}-z$ is somewhat uncertain.

The total VdW force $F_{W}$ and its gradient are calculated by differentiating Eq. (3) with respect to $z_{j}$. Calculated results are shown in Fig. 3. For the hemispherical geometry, the VdW force and its gradient are significant compared to the corresponding short-range counterparts per tip atom (Fig. 2). The VdW force increases roughly proportional to the assumed radius $r \gg z_{j}$ in the range $200-1000 \AA$, typical for the end of a carefully etched support tip used in STM or combined STM/SFM investigations. Even larger VdW forces are expected for flat-ended cylindrical tips. ${ }^{24}$ These results explain in part why Goodman and Garcia ${ }^{23}$ found VdW forces and gradients of much larger magnitude for different materials assuming a spherical tip of diameter $2000 \AA$ at $z_{j}=10 \AA$. On the other hand, for a sharp conical tip with $\alpha<45^{\circ}$, the VdW force is less than $0.1 \mathrm{nN}$, while the force gradient is in the range of $0.1 \mathrm{~N} / \mathrm{m}$. By contrast, the gradient of the short-range force in the attractive range is $\sim 1 \mathrm{~N} / \mathrm{m}$. These estimates are similar to those of Dürig and co-workers. ${ }^{17}$ The long-range $\mathrm{VdW}$ force is strongly shape dependent. For a realistic support tip, which is unavoidably rounded near its apex and becomes approximately conical further away, the front part will determine the net VdW interaction as long as $z_{j} \ll r$. As a result the corresponding force and to a lesser extent its gradient can remain significant even at small separations where they are overestimated by the present calculations.

\section{ATOM TRANSFER}

An atom at the apex of a sharp tip, just like an adsorbed host atom on a flat surface, has a smaller coordination and thus weaker binding as compared to that in the bulk. As the tip approaches the sample, the apex atom is attracted to the sample. To picture what can happen, two interaction energy curves $E_{i}(z)$ like those in Fig. 1 can be thought of as attached to each electrode in opposite directions and superposed. The resulting curve approximately represents the interaction energy of an atom between two electrodes as a function of its coordinate $z$ measured with respect to the left electrode. It rises to large values in the repulsive range at small $z$ and exhibits a single minimum at $z \simeq s / 2$ ( $s$ is the distance between the two surfaces) for $s<2 z_{m}$. Otherwise one obtains two minima separated by an energy barrier. The evolution of the interaction energy versus position $z$ of an $\mathrm{Al}$ atom between $H$ sites of two $\mathrm{Al}(001)$ surfaces is presented in Fig. 4 for several separations. These results were obtained from computations analogous to those described in Sec. II, but with lateral $(3 \times 3)$ periodicity of the $\mathrm{Al}$ atom facing the $H$ sites of two $\mathrm{Al}(001)$ surfaces. Since the lateral positions of the $\mathrm{Al}$ atom relative to both slabs are identical, the resulting curves are symmetric. Because interactions with, as well as between, both electrodes are included, the actual interaction energy deviates from $E_{i}(z)+E_{i}(s-z)$, with $E_{i}(z)$ calculated for an atom interacting with a single electrode only. The energy barrier decreases as the separation between electrodes is

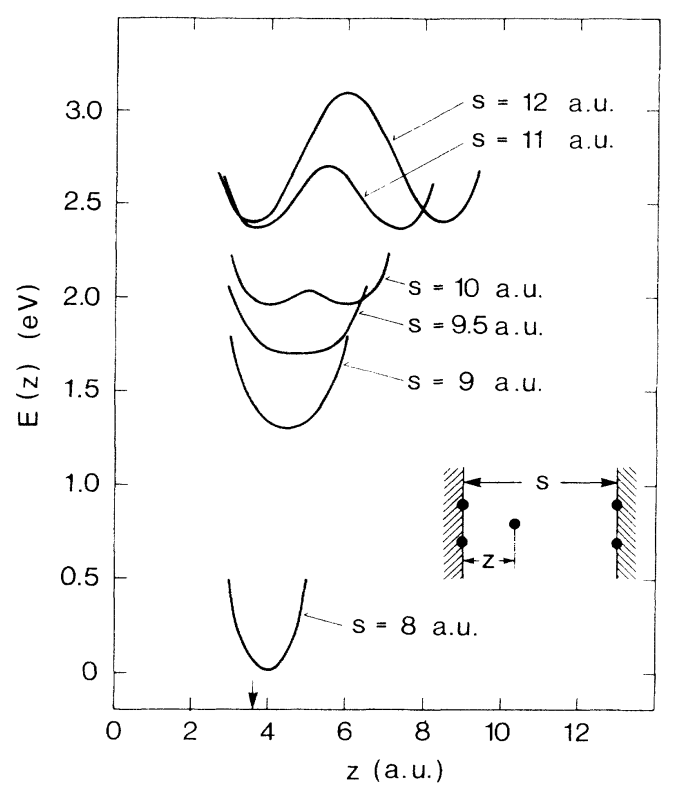

FIG. 4. Energy of a single $\mathrm{Al}$ atom between two $\mathrm{Al}(001)$ slabs versus its distance from the left electrode (slab) as described in the inset. The single $\mathrm{Al}$ atom faces the $H$ site of both surfaces. The zero of energy is taken at the minimum of $E(z)$ corresponding to $s=8$ a.u.

decreased and hence the rate at which the apex atom can hop between the stable minima on each side by thermal activation or by tunneling increases exponentially. This can in principle become observable before the minima merge into a single one. ${ }^{31}$ In the course of approaching the sample the distance of the apex atom from the rest of the tip gradually increases owing to increasing attraction to the sample. Moreover, the minima become shallower and the barrier becomes lower and narrower as one would infer from $E_{i}(z)+E_{i}(s-z)$. As a consequence, hopping sets in at a larger separation. As the barrier collapses upon further approach, the distance of the apex atom from either electrode is larger than that corresponding to the equilibrium binding to only one electrode. These effects are amplified if both the tip and sample are allowed to deform, in partial analogy to the avalanche effect ${ }^{32,33}$ in which atomic layers of two interacting semi-infinite slabs collapse when the spacing between them falls below a critical distance leading to a discontinuous drop in the interaction energy. In the present computations the tip (except the outermost atom) and sample are rigid, so that the interlayer separations are fixed.

A concomitant effect dramatically evidenced by comparing Figs. 1 and 4 is also initiated by attraction of the atom to both electrodes. After the barrier disappears, the atom is stable in the middle with a considerably larger binding energy at a significantly larger separation than twice the corresponding quantities with only one electrode present. This means that the atom can gain an additional stabilization energy between two electrodes. All these phenomena have several important implications 
in SFM. (i) Upon approach the outermost atoms of the tip (or adatoms on the sample) will hop to the other side at a rate proportional to $\exp \left[-Q_{ \pm}(s) / k_{B} T\right]$. Here $Q_{ \pm}$ is the activation energy for transfer over the barrier in the two opposite directions. Atom transfer via tunneling through the barrier can in principle also contribute at sufficiently low temperature, but such a process can be meaningful only for very low barriers, i.e., in a narrow range of separations just prior to barrier collapse, and will therefore be very difficult to identify. The difference between $Q_{+}$and $Q_{-}$comes from the asymmetry of the interaction energy and will generally occur owing to several reasons. First of all, the tip and sample are usually made of different materials. The tip is usually chosen to be a hard material like W or Si (for SFM). Even if the tip and sample consist of, or are coated with, the same material, their shapes are different. An atom is then likely to prefer a site of maximum coordination, i.e., at a step or kink on the sample side. That the stronger binding in Fig. 1 occurs for an atom facing the $H$ site is in accordance with this picture. For such an asymmetric interaction energy, the time spent by an atom bound in the deeper minimum will be much longer, and the probability of the transfer of atoms towards the deeper minimum will be much higher. (ii) Although the avalanche process might reduce to single-atom transfer if the tip apex is sharp, and if both sample and tip are sufficiently hard, the combined effects of thermal activation and softening can induce more extensive wetting of one electrode by the other. ${ }^{34}$ (iii) Even if the barrier energy $\left(Q_{+}\right.$or $\left.Q_{-}\right)$is large for a given condition, it can be momentarily lowered by an external agent. For instance, as in field desorption, ${ }^{31,35}$ the quasisymmetric position of energy minima can be modified in such a way that atom transfer to one side will be enhanced. An external field of $\sim 1$ V/ $\AA$ can achieve this, since not only can it more easily penetrate the apex of the tip with its lower coordination, it can also penetrate into the top layer of a metal. ${ }^{35}$ As a result of the controlled lateral and perpendicular motion of the tip under an appropriate voltage, adsorbed atoms can, for instance, be relocated to desired positions. ${ }^{35-37}$ In the presence of a sharp tip, the increased binding of an atom at a single central minimum below a certain tip-sample separation, as in Fig. 4, will be limited to the vicinity of the tip, thus enabling controlled lateral relocation independent of voltage. ${ }^{38}$ The observation that atom transfer changes direction with the current independent of voltage has been attributed to the adatom excitation (heating) by tunneling electrons. (iv) The configuration with an atom between two electrodes also facilitates the collapse of the potential barrier between two electrodes below $E_{F}$. This effect, not to be confused with the collapse of the barrier for atom transfer, occurs at much larger separations. As mentioned in our discussion of Fig. 2, the collapse in question occurs for $z \lesssim 9$ a.u. in the case of two $\mathrm{Al}(001)$ surface. With an atom in between this should occur below an interelectrode separation slightly beyond the range of $s$ covered in Fig. 4. At smaller separations we expect coupled electronic and mechanical changes similar to those obtained in earlier studies. ${ }^{6,8,39-45}$ Since the apparent maximum of the po- tential along the $z$ axis through the atom is actually a saddle point in three dimensions, the classically allowed channel which forms does not allow electron wave propagation until $s$ is reduced such that the electron density rises in the channel. This occurs quite rapidly, resulting in bond formation at a slightly smaller separation. Judging from the sudden increase in binding below $s_{c} \simeq 9.5$ a.u. in Fig. 4, we estimate this to be also the critical value for electrical contact. Because the atom is then stable in the middle, waveguidelike channels actually exist on both sides. When the tip and sample are brought closer together, new conduction channels can open and the character of electron transport should change from tunneling to ballistic. ${ }^{40}$

Eventually mechanical contact is initiated; attraction quickly changes to repulsion leading to plastic deformations and to jumplike increase in contact area ${ }^{32-34}$ and corresponding changes in conductance. ${ }^{40}$ Different regimes (i.e., conventional tunneling, electronic contact, and mechanical contact) in the operation of STM were treated earlier. ${ }^{6,41}$ Between $s \simeq 13$ and 9 a.u. the lateral size of the channel ${ }^{39,40,42-44}$ is not sufficient per se to open the lowest ballistic propagation mode with a quantum conductance $2 e^{2} / h$. Even so, the atom between both electrodes can have a resonance state near the Fermi energy, ${ }^{8}$ which can raise the conductance to that level. This situation is reminiscent of a double-barrier quantum well, and gives rise to an increased conductivity even if coupling to electrodes is via evanescent waves. The same situation was found earlier for states bound to a quantum dot or an impurity in a one-dimensional (1D) mesoscopic channel. ${ }^{46}$ Of course, the resonance condition will be modulated depending on separation and lateral position as the tip is scanned above the sample surface. The resulting modulation of the conductance, owing to changes in the potential barrier and in the wave functions of relevant states, is either purely electronic ${ }^{8}$ or induced by displacement of the atom ${ }^{46}$ (likely a combination of these effects), and offers a likely explanation of the anomalously large corrugation observed in STM of the close-packed surfaces with a nominally very flat electron density profile, such as $\mathrm{Al}(111) .{ }^{47} \mathrm{~A}$ full theory incorporating the position-dependent self-consistent potential is, to our knowledge, still lacking. If $s$ is sufficiently large compared to $s_{c}$, the appropriate configuration is the initial one (i.e., one of the minima in the interaction energy curve), which may be metastable. As $s$ approaches $s_{c}$, the atom eventually hops back and forth between alternative positions many times during a measurement, and the tunneling current must be a weighted average which accounts for the dwell time and thermal motion around each minimum. Close to $s_{c}$ this motion becomes more extensive and anharmonic. The corresponding changes in the current power spectrum are worth investigating. Finally note that if the interaction energy curve is symmetric as in Fig. 4, these motional averaging effects are enhanced. This situation, which also favors resonant tunneling with maximum transmission when $s \sim s_{c}$, can arise when sample atoms have previously been transferred to the tip [as-occurred in the STM study of $\mathrm{Al}(111)$ (Ref. 47)]. 


\section{FORCE AND CONDUCTIVITY}

Going back to Secs. I and II it is clear that the (shortrange) electron-mediated tip-sample interaction arises because the potential barrier between electrodes allows wave functions to overlap. According to Bardeen's perturbation theory of tunneling, ${ }^{48-50}$ the conductance is also determined by the same overlap of wave functions at the Fermi level. Therefore, in the attractive range it is expected that force and conductance are interrelated. Earlier, we pointed out the reversible modifications of electronic states prior to contact, and correlations between perpendicular force $F_{s \perp}$ and barrier height $\phi_{B} \cdot{ }^{7,8}$ Denoting the transfer-matrix element between the tip $\left(\Psi_{T}\right)$ and sample $\left(\Psi_{S}\right)$ wave functions with respect to the combined tip-sample Hamiltonian as $U_{T, S}(z)=$ $-\left\langle\Psi_{S}\left|H_{T+S}(z)\right| \Psi_{T}\right\rangle$, the shifts of the corresponding energies $\epsilon_{T}$ and $\epsilon_{S}$ can be expressed in first-order perturbation theory. Experimentally, Dürig et al. ${ }^{17}$ drew attention to the correlation between force gradient and tunneling conductance $G$ in the course of tip approach. More recently Chen ${ }^{50}$ concluded that the force should be approximately proportional to the square root of the conductance. First he related the interaction energy to the splitting of coupled states via $\sum_{T, S} U_{T, S}(z)$. On the other hand, $U_{T, S}(z)$ itself is approximately equal ${ }^{52}$ to the tunneling matrix element $M_{T, S}(z)$. As a result, $E_{i}(z) \simeq \sum_{T, S} M_{T, S}(z)$, and hence the perpendicular component of the electron-mediated force can be extracted as $F_{s \perp} \simeq-\sum_{T, S} \partial M_{T, S} / \partial z$. If, as assumed by Chen, a resonance near the Fermi energy localized at the apex of the tip singles out a dominant $M_{T, S}$, this leads to $F_{s \perp}=\xi \kappa \sqrt{G}$, since the tunneling conductance ${ }^{48}$

$$
G \propto \sum_{T, S}\left|M_{T, S}(z)\right|^{2} \delta\left(\epsilon_{T}-E_{F}\right) \delta\left(\epsilon_{S}-E_{F}\right)
$$

then decays exponentially with tip-sample separation $z$. Although only states at the Fermi level contribute to $G$, states below $E_{F}$ (which, in turn, decay faster) are also involved with perpendicular force. In the typical range of STM or combined STM/SFM operation, covering 2$3 \AA$ in $z$ (Refs. 17 and 51) beyond electrical contact, we therefore expect $F_{s \perp}$ to decay faster than $\sqrt{G}$. This has, in fact, been observed by Dürig and co-workers, ${ }^{17}$ who obtained good fits to the Rydberg function with $\lambda \sim 0.42 \AA$ for their data on $\mathrm{Ir}$, and $\lambda \simeq 0.66 \AA$ for our results for $\mathrm{Al}(111)$ (Ref. 8) as compared to $\kappa^{-1} \sim 1 \AA$. Furthermore, in this range the maximum height $\phi_{B}$ of the potential barrier is considerably depressed below its asymptotic value $\phi$ (the average of the work functions of sample and tip) and changes with $z$ together with the barrier profile. Although the latter effect conspires to produce an apparent barrier height ${ }^{43} \phi_{A}>\phi_{B}$ which remains finite almost down to the point of electrical contact, $\phi_{A}$ has also been predicted to be depressed. ${ }^{43,46}$ Its slow $z$ dependence may be difficult to detect over the limited experimental range, however. Finally the microscopic expressions for the interaction energy and total force cannot in general be written in terms of shifts and splittings of the occupied electronic eigenvalues induced by a weak interaction between two subsystems. ${ }^{53}$ The only exception is the special case where one of the subsystems has no valence states (e.g., rare-gas atoms) and the Hartree-Fock approximation is used. For metallic or other subsystems with a short-range interaction due to overlap of their valence states, the LDA is a better approximation. Although the leading asymptotic term is then in fact proportional to the sum of the eigenvalue shifts, the next contribution from exchange remains important. ${ }^{53}$ For all these reasons one cannot expect the relation between $F_{s \perp}$, conductance $G$, and $\kappa$ to be as simple as Chen's. Nevertheless, some relation is expected to exist as long as $\phi_{A}>0$, and should approach Chen's as $z$ is increased beyond the point where $\phi_{B}$ becomes positive.

In order to test the validity of Chen's argument, we first seek a similar relation between the force $F_{s \perp}$ calculated in Sec. II and the maximum barrier height $\phi_{B}$. For $\phi_{B}$ we take the difference between the maximum of the planar average of our calculated SCF potential at $z / 2$ and $E_{F}$. The plot in Fig. 5 indicates that the short-range force is in fact (calculated in the range where $F_{s \perp}$ is essentially site independent and also $\phi_{B}>0$ ) proportional to $\kappa \exp (-\kappa z)$, where $\kappa=\sqrt{\phi_{B}}$ in a.u. It is important to realize that $\phi_{B}$ remains quite low, so that $\kappa^{-1}$ varies between 2.5 and 5.3 a.u. in the limited range studied, where a comparison with our previous computations appears meaningful. Moreover, it extends somewhat beyond the range where Dürig and co-workers ${ }^{17}$ as well as we (see inset to Fig. 1) obtained close fits to the Rydberg function with a decay length $\lambda \sim 1$ a.u. This indicates that quite different effective decay lengths can be obtained from fits over a limited range to different functions containing a decaying exponential whenever the latter is not sufficiently small. Furthermore, a confinement effect analogous to that found for a protruding tip atom ${ }^{8}$ may be significant, especially at the lower limit of $z$ for the

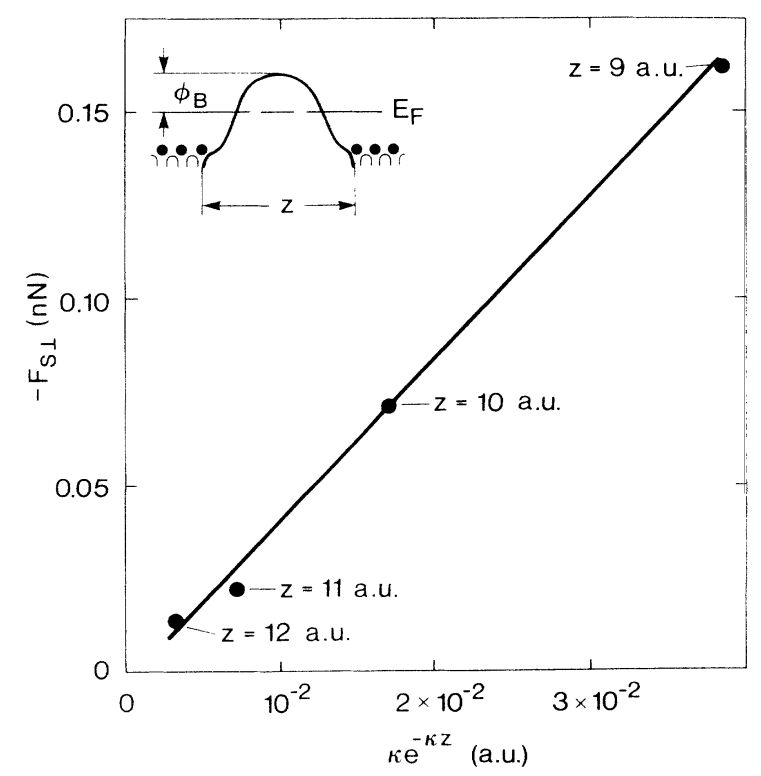

FIG. 5. Calculated perpendicular component of the (short-range) force $F_{s \perp}(z)$ versus $\kappa \exp (-\kappa z)$ for separations $z=9,10,11$, and 12 a.u. 
$T$-site geometry. Indeed, the potential $V(\mathbf{r})$ has significant lateral variation especially close to the corrugated electrode. Therefore, $\phi_{B}$ is obtained from the potential at $z / 2$ for the $H$-site geometry, which exhibits a weaker $x, y$ dependence. In view of all these qualifications, the proportionality apparent in Fig. 5, although suggestive, must be viewed with caution.

Owing to the artificial periodic boundary conditions imposed in supercell SCF calculations and the resulting coarse discretization in $\mathbf{k}$ space, a calculation of the conductance is tedious. In order to avoid complications arising from the $x, y$ dependence of the potential, and to explore the relation between short-range force and conductance over a wider range, we now resort to the jellium model following the variational treatment originally applied to determine the interaction between two flat metal surfaces. ${ }^{54}$ The electronic charge density of the semi-infinite left electrode is parametrized as $\rho_{L}\left(z^{\prime}\right)=\rho_{+}\left[1-\exp \left(\beta z^{\prime}\right) / 2\right]$ for $z^{\prime}<0$ in the positive background region and $\rho_{L}\left(z^{\prime}\right)=\rho_{+} \exp \left(-\beta z^{\prime}\right) / 2$ for $z^{\prime}>0$ in the vacuum region. Here $\rho_{+}$is the uniform charge density corresponding to $\mathrm{Al}$, and $z^{\prime}=0$ marks the jellium edge, i.e., $z=d_{0} / 2$ in our previous notation. The charge density $\rho_{R}\left(z^{\prime}\right)$ of the right electrode is given by the same expressions with $z^{\prime}$ replaced by $z_{j}-z^{\prime}, z_{j}$ being the separation between jellium edges. That the charge density is expressed in terms of a single exponent is of course an approximation, but makes the problem easily tractable. In density-functional theory, ${ }^{55}$ the ground-state energy of a confined interacting electron gas is expressed as a functional of the electron number density $\rho(\mathbf{r})$, which should be minimized for the correct $\rho(\mathbf{r})$. In the present study we assume that the electron density of the coupled system can be represented as $\rho_{L}+\rho_{R}$ and computed the energy functional with local exchange and correlation potential as in Ref. 54. In contrast to that work, we minimized the functional for each separation, thus obtaining $\beta\left(z_{j}\right)$. In this way the modification of the electronic states due to electrode-electrode (or tipsample) interaction is taken into account to some extent and the resulting interaction energy is closer to the selfconsistent LDA jellium calculations performed later. ${ }^{56}$

In Fig. 6 we first examine the validity of this variational jellium approach that expresses the charge density between two identical flat parallel electrodes in terms of a single optimized decay constant $\beta\left(z_{j}\right)$. First, we compare the corresponding potential with the planar average of the SCF pseudopotential calculated for the $H$-site geometry for $z_{j}=7.2$ a.u. and $z=11$ a.u., respectively, in Fig. 6(a). Second, the variation of $\phi_{B}$ with electrode separation obtained from these calculations is compared in Fig. 6(b). Finally, we compare $F_{s \perp}\left(z_{j}\right)$ calculated in the jellium model with that calculated in Sec. II at the $H$ site in Fig. 6(c). For the sake of direct comparison the distance $z$ between the outermost layers of the slabs is expressed in terms of $z_{j}=z-d_{0}$. Quantities calculated for the $H$ site are compared with the corresponding jellium results, because this geometry corresponds to the correct registry for adhesion at $z_{j}=0$. Besides, as mentioned in our earlier discussion about $\phi_{B}$, the three-dimensional
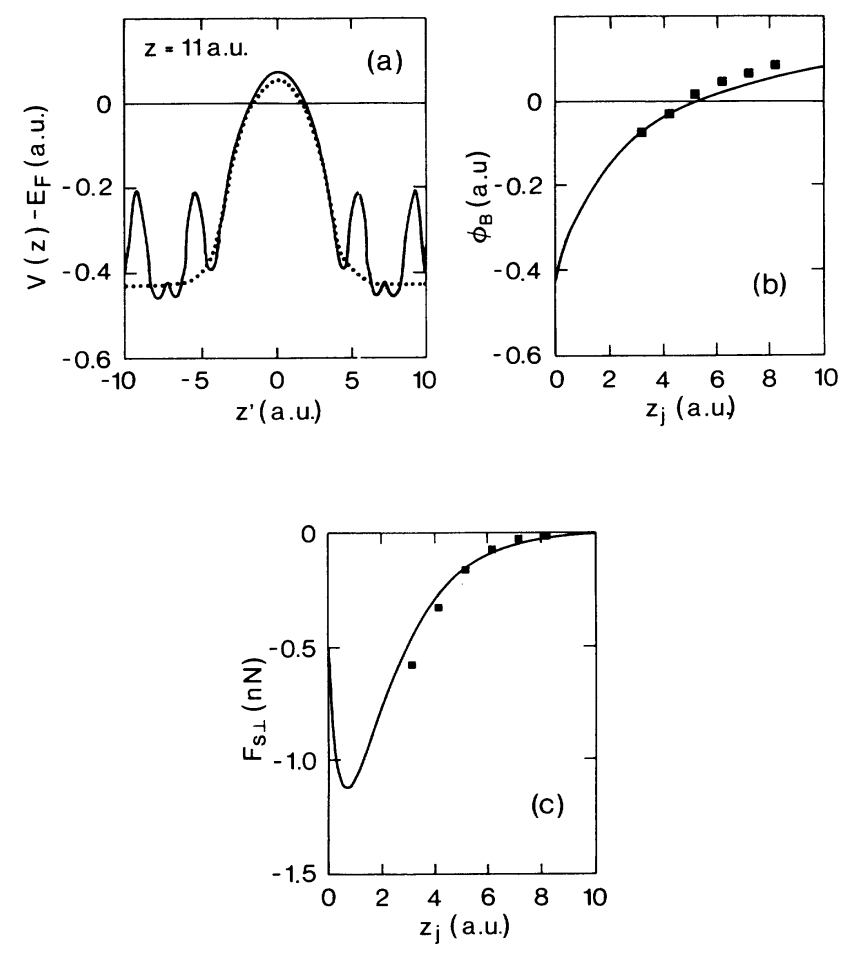

FIG. 6. (a) Potential energy $V\left(z^{\prime}\right)$ relative to the Fermi level $E_{F}$ for $z=11$ a.u.; the full curve corresponds to a planar averaged SCF pseudopotential, the dotted curve is obtained from the jellium calculations with $z_{j} \simeq 7.2$ a.u. (b) Potential barrier $\phi_{B}$ versus separation $z_{j}$ between jellium edges; the full curve is our jellium approximation, squares are SCF results. (c) Perpendicular force versus separation as in (b).

SCF pseudopotential then has a weaker $x, y$ dependence in the middle of the barrier. As indicated in connection with Fig. 2, force curves from our SCF calculations exhibit a significant site dependence in the range where $\phi_{B}<0$. Actually, the barrier height calculated from the planar average of the SCF pseudopotential for the $T$ site is consistently higher than that for the $H$ site because the higher potential between $H$ sites facing each other in the former geometry contributes more to the average. The difference persists even if $\phi_{B}>0$, becoming small at large $z\left(\phi_{B}^{T}-\phi_{B}^{H}=0.2 \mathrm{eV}\right.$ for $z=11$ a.u.), and large close to the point where $\phi_{B}=0\left(\phi_{B}^{T}-\phi_{B}^{H}=0.6 \mathrm{eV}\right.$ for $z=9$ a.u.). In spite of all these reasons for deviations between the results of our two quite different approaches, the level of agreement seen in Figs. 6(a)-6(c) is remarkable. For $z<9$ a.u. $\left(z_{j}<5.2\right.$ a.u.) the magnitudes of the attractive forces from our SCF pseudopotential calculations systematically exceed those obtained from our jellium approximation. This is related to the collapse of the potential barrier below $E_{F}$, which makes self-consistency and the discrete atomic structure of electrodes particularly important. Nevertheless, our jellium approach yields a very reasonable force curve. In particular, $F_{s \perp}$ almost vanishes at $z_{j}=0$, as it should and also does in a self-consistent treatment, ${ }^{56}$ in contrast to the original work where $\beta$ was kept equal to the optimum value for a single electrode. ${ }^{54}$ Even for large separations 
( $z \gtrsim 12$ a.u.) both approaches show that $\phi_{B}$ still varies appreciably, so that the decaying tails of the wave functions at $E_{F}$, and hence the charge density, cannot be well represented by a single decay constant. As a matter of fact, at large separation SCF slab calculations in a supercell also fail to give an accurate representation of such tails because of the truncated plane-wave basis set. Nevertheless, the jellium approach described above provides a simple and reasonably accurate way of calculating the interaction energy, force, and potential between two flat simple metal electrodes in a broader range than much more demanding SCF pseudopotential calculations.

Having tested our variational jellium approximation, we next consider the corresponding transmission properties. To this end we first compare the transmission probability $T$ of an electron tunneling at the Fermi level through the potential barrier between two electrodes as a function of $z_{j}$ calculated essentially exactly using the transfer-matrix method ${ }^{46}$ and in the WentzelKramers-Brillouin (WKB) approximation for an opaque barrier. ${ }^{57}$ We also consider the quantities $\exp \left(-2 \kappa z_{j}\right)$ and $\exp \left(-2 \kappa_{0} z_{j}\right)\left(\kappa_{0}\right.$ corresponding to the barrier height at infinite separation) which, strictly speaking, govern transmission through a barrier of constant height, but are often used to extract an apparent barrier height from an exponential fit to conductance versus separation. Figure 7 compares the variation of these quantities as functions of $z_{j}$. The transmission probability calculated exactly becomes unity when $z_{j}=0$ (i.e., when two jelliums merge), and so does $\exp \left(-2 \kappa_{0} z_{j}\right)$ for a mathematical reason. However, both $T(\mathrm{WKB})$ and $\exp \left(-2 \kappa z_{j}\right)$ reach unity earlier, i.e., when the barrier collapses for $z_{j} \lesssim 5$ a.u. One interesting conclusion from Fig. 7 is that the $T$ (WKB) overestimates the exact transmission probability, but stays reasonably close at large separations, as expected. In fact, for sufficiently large separations all quantities shown in Fig. 7 become proportional since the underlying potential barrier is rather flat and wide. For small $z_{j}$, on the other hand, all approximate expressions

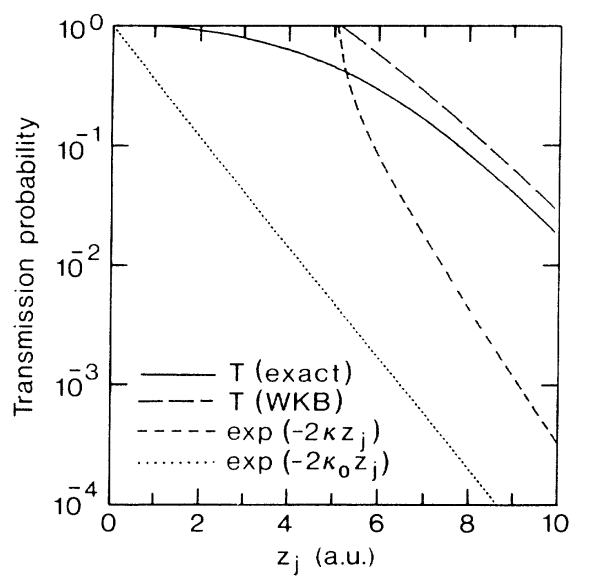

FIG. 7. Transmission probability at $E_{F}$ versus separation $z_{j}$ between jellium edges. The dependence emerging from an accurate computation (full curve) is compared with various approximations. For details refer to the text. fail to give good estimates for the transmission probability. This statement also applies for the original and modified forms of the transfer Hamiltonian approach. ${ }^{58}$ The quantity $\exp \left(-2 \kappa z_{j}\right)$, which gives rise to the simple dependence apparent in Fig. 5, poorly reflects the $z$ dependence of the transmission. This can be traced back to the changing barrier shape which cannot be described by its maximum height alone. ${ }^{43}$ Integrating the exact transmission over transverse momenta, keeping $E=E_{F}$, we obtain a conductance curve (not shown) with the same appearance lying closer to $\exp \left(-2 \kappa_{0} z_{j}\right)$.

Figures $8(\mathrm{a})-8(\mathrm{~d})$ display how the short-range force and the conductance or various quantities related to the transmission probability $T$ at $E_{F}$ are related within our jellium model. It is important to clarify some aspects related to the trends apparent in this figure, as compared to Fig. 5. First, Figs. 8(a)-8(d) span a wider and different range of separation, which extends beyond the range included in Fig. 5, especially Figs. 8(c) and 8(d). However, the range must still be limited because it is not appropriate to represent the charge density throughout the barrier by a single $\beta\left(z_{j}\right)$ for either very small or very large separations. Also, as seen in Figs. 6(a) and 6(b), the potential barrier is slightly underestimated as compared to our SCF results. Finally, for large separations $F_{s \perp}$ becomes so small that it is swamped by the VdW contribution, which is absent in the LDA. Hence only a portion of the curves depicted in Figs. 5 and 8 may be relevant for a meaningful comparison.

In Fig. 8(a) the plot of perpendicular force versus $\kappa \exp \left(-\kappa z_{j}\right)$ once again indicates a simple relation in the
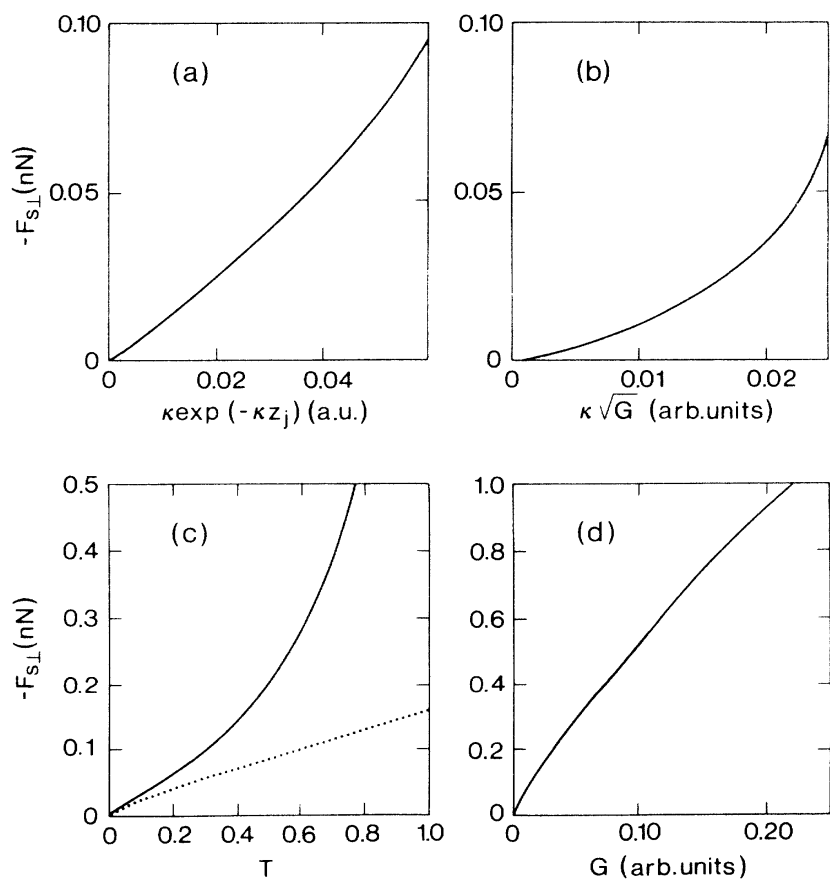

FIG. 8. Perpendicular force versus (a) $\kappa \exp \left(-\kappa z_{j}\right)$ as in Fig. 5 , (b) $\kappa \sqrt{G}$, (c) transmission probability $T$ calculated exactly (full curve) and using the WKB approximation (dotted curve), (d) conductance $G$. All calculated within the jellium approximation. 
weak-attractive force range. However, this relation is approximately linear only in a limited range where the attractive force is very weak. Since $\exp \left(-2 \kappa z_{j}\right)$ is a poor approximation to the exact transmission probability $T$ or to the conductance $G$, it is not surprising that plots of force versus $\kappa \sqrt{G}$ or versus $T$ in Fig. 8(c) fail to reveal any proportionality, and by the same token do not support a relation of the type proposed by Chen, ${ }^{50}$ except perhans very close to the origin (i.e., for very large separations) in Fig. 8(b). On the other hand, approximate linear relations, albeit over different limited ranges, are found between $F_{s \perp}$ and the WKB approximation for $T$ in Fig. 8(c), and the exact conductance $G$ in Fig. 8(d). Taken together, these plots demonstrate that there is no simple relation between force and any quantity related to the exact transmission or conductance over the range of $z_{j}$ spanned in Figs. 8(b)-8(d). Although our jellium calculations confirm the approximate proportionality between the short-range force and the quantity $\kappa \exp (-\kappa z)$ related to the maximum average barrier height pointed out earlier ${ }^{10}$ and in Fig. 5, it appears to have less physical significance than the fit to the Rydberg function discussed in Sec. II, just like the other approximate linear relations described above. Keeping in mind all the approximations involved in the jellium model as well as Chen's arguments, we can only expect a trend rather than an exact and universal relation. In actual STM/SFM experiments deviations from any universal expression are expected depending on the measurement conditions. For example, the structure of the tip enters because it produces a confinement effect and also affects the contribution of the VdW force. Theoretical results obtained by different approximate methods can also differ in properly representing the shape of the potential barrier.

\section{SUMMARY}

In this work we investigated different manifestations of the interaction between two rigid $\mathrm{Al}(001)$ slabs for different separations using the SCF pseudopotential method. We calculated the interaction energy, perpendicular, and lateral forces acting on one slab for several relative position of the other slab. The interaction energy of a single $\mathrm{Al}$ atom located between $H$ sites of such two slabs has also been studied. Similar physical quantities, as well as the transmission probability and conductance between two electrodes, have also been calculated within a variational jellium approximation. Our results are relevant for the interpretation of SFM experiments with a blunt tip and their correlation with simultaneous STM measurements on metals. The important aspects of the work can be summarized as follows. (i) The interaction energy and the short-range perpendicular force are site dependent, but both can be accurately represented at different sites by a Rydberg function in terms of differently scaled energy but with the same decay length $\lambda \simeq 1$ a.u. (ii) Calculated force curves indicate a corrugation of 1.2 a.u. for constant perpendicular load in the range of $\pm 1 \mathrm{nN} /$ atom. (iii) With an extra atom in between, a crossover between an energy dependence with two minima to one with one much deeper minimum in the center is found for a slab separation of $\simeq 2.5$ interlayer spacings. This observation is relevant for controlled atom transfer experiments. (iv) The net Van der Waals force and its gradient can be important at small tip-sample separation if the support tip has a radius of curvature as small as $200 \AA$. However, they have negligible effects for a sharp conical tip. (v) Although the short-range perpendicular force turns out to be proportional to a simple quantity related to the maximum height of the tunneling barrier in the manner suggested by Chen, no simple relation is found with the conductance or the transmission probability at the Fermi energy calculated within our jellium approximation.

\section{ACKNOWLEDGMENTS}

This work is partially supported by the Joint Project Agreement between Bilkent University and IBM Zurich Research Laboratory. The authors wish to thank Dr. E. P. Stoll for valuable assistance and Dr. U. Dürig for stimulating discussions. ${ }^{*}$ Permanent address: Department of Physics, Bilkent Uni-
versity, Bilkent 06533, Ankara, Turkey.
${ }^{1}$ G. Binnig, C. F. Quate, and Ch. Gerber, Phys. Rev. Lett.
56, 930 (1986).
${ }^{2}$ G. Binnig, Ch. Gerber, E. Stoll, T. R. Albrecht, and C. F.
Quate, Europhys. Lett. 3, 1281 (1987); T. R. Albrecht and
C. F. Quate, J. Vac. Sci. Technol. A 6, 271 (1988); R. Er-
landsson, G. M. McClelland, C. M. Mate, and S. Chiang,
ibid. 6, 266 (1988); H. Heinzelmann, E. Meier, P. Grütter,
H.-R. Hidber, L. Rosenthaler, and H.-J. Güntherodt, ibid.
6, 275 (1988); O. Marti, B. Drake, S. Gould, and P. K.
Hansma, ibid. 6, 287 (1988).
${ }^{3}$ G. Binnig, H. Rohrer, Ch. Gerber, and E. Weibel, Phys.
Rev. Lett. 49, 57 (1982); G. Binnig and H. Rohrer, Rev.
Mod. Phys. 59, 615 (1987).
${ }^{4}$ J. M. Soler, A. M. Baro, N. Garcia, and H. Rohrer, Phys.
Rev. Lett. 57, 444 (1986).

${ }^{5}$ U. Dürig, J. K. Gimzewski, and D. W. Pohl, Phys. Rev. Lett. 57, 2403 (1986); H. Yamada, T. Fujii, and K. Nakayama, J. Vac. Sci. Technol. A 6, 293 (1988).

${ }^{6}$ S. Ciraci and I. P. Batra, Phys. Rev. B 36, 6194 (1987); E. Tekman and S. Ciraci, ibid. 40, 10286 (1989); S. Ciraci, in Basic Concepts and Applications of Scanning Tunneling Microscopy and Related Techniques, edited by $\mathrm{H}$. Rohrer, N. Garcia and J. Behm (Kluwer, Amsterdam, 1990), p. 119. ${ }^{7}$ S. Ciraci, A. Baratoff, and I. P. Batra, Phys. Rev. B 41, 2763 (1990).

${ }^{8}$ S. Ciraci, A. Baratoff, and I. P. Batra, Phys. Rev. B 42, 7168 (1990).

${ }^{9}$ These state-of-the-art total-energy calculations were performed in momentum space using normconserving Hamann-Schlüter-Chiang pseudopotentials and 
the Ceperley-Alder exchange-correlation potential. For further details see Refs. 7 and 8.

${ }^{10} \mathrm{~S}$. Ciraci, E. Tekman, M. Gökçedağ, I. P. Batra, and A. Baratoff, Ultramicroscopy 42-44, 163 (1992).

${ }^{11}$ The interaction energy is given by $E_{i}(z)=E_{S+T}(z)-E_{S}-$ $E_{T}$ in terms of the total energies of the tip and sample together $\left[E_{S+T}(z)\right]$, sample $\left[E_{S}\right]$ and tip slab $\left[E_{T}\right]$ alone, each calculated separately in the same tetragonal supercell of dimensions $R_{1}=R_{2}=5.4$ a.u., $R_{3}=46$ a.u.

${ }^{12} \mathrm{By}$ definition $E_{i}(z)<0$ indicates an attractive interaction energy, and the maximum of the adhesion energy or surface energy is half of the binding energy between semi-infinite crystals. Our unit of binding energy is given for the unit cell in Ref. 11, which includes energy of both surfaces.

${ }^{13}$ S. Ciraci and I. P. Batra, Phys. Rev. B 36, 6194 (1987); see also I. P. Batra, S. Ciraci, G. P. Srivastava, J. S. Nelson, and C. Y. Fong, ibid. 34, 8246 (1986).

${ }^{14}$ J. H. Rose, J. Ferrante, and J. R. Smith, Phys. Rev. Lett. 47, 675 (1980); Phys. Rev. B 28, 1835 (1983); A. Banerjea and J. R. Smith, ibid. 37, 6632 (1988).

${ }^{15}$ J. R. Smith, and A. Banerjea, Phys. Rev. Lett. 59, 2451 (1987).

${ }^{16}$ A. Banerjea, J. R. Smith, and J. Ferrante, J. Phys. Condens. Matter 2, 8841 (1990).

${ }^{17}$ U. Dürig, O. Züger, and D. W. Pohl, Phys. Rev. Lett. 65, 349 (1990); U. Dürig and O. Züger, Vacuum 41, 382 (1990); and (unpublished).

${ }^{18} \mathrm{H}$. Helmann, Einführung in die Quanten Theorie (Deuticke, Leipzig, 1937); R. P. Feynman, Phys. Rev. 56, 340 (1939); J. C. Slater, J. Chem. Phys. 57, 2389 (1972).

${ }^{19}$ C. M. Mate, G. M. McCleland, R. Erlandson, and S. Chiang, Phys. Rev. Lett. 591942 (1987).

${ }^{20}$ W. Zhong and D. Tomanek, Phys. Rev. Lett. 64, 3054 (1990).

${ }^{21}$ J. B. Sokoloff, Phys. Rev. Lett. 66, 965 (1991).

${ }^{22}$ C. Girard, D. Van Labeke, and J. M. Vigoureux, Phys. Rev. B 40, 12133 (1989); C. Girard, ibid. 43, 8822 (1991).

${ }^{23}$ F. O. Goodman and N. Garcia, Phys. Rev. B 43, 4728 (1991).

${ }^{24}$ U. Hartmann, Phys. Rev. B 43, 2404 (1991).

${ }^{25}$ E. M. Lifshitz, Zh. Eksp. Teor. Fiz. 29, 94 (1956) [Sov. Phys. JETP 2, 73 (1956)].

${ }^{26}$ J. N. Israelachvili, Intermolecular and Surface Forces (Academic, London, 1985), Chap. 11.

${ }^{27}$ J. E. Inglesfield and E. Wikborg, J. Phys. F 5, 1475 (1975); J. E. Inglesfield, ibid. 6, 687 (1976).

${ }^{28}$ E. Zaremba and W. Kohn, Phys. Rev. B 13, 2270 (1976).

${ }^{29}$ B. N. J. Persson and E. Zaremba, Phys. Rev. B 30, 5669 (1984); A. Liebsch; ibid. 33, 7248 (1986).

${ }^{30}$ P. Nordlander and J. Harris, J. Phys. C 17, 1141 (1984); J. F. Annet and P. M. Echenique, Phys. Rev. B 34, 6853 (1986).

${ }^{31}$ R. Gomer, IBM J. Res. Develop. 30, 426 (1986).

${ }^{32}$ J. B. Pethica and A. P. Sutton, J. Vac. Sci. Technol. A 6,
2490 (1988); J. Phys. Condens. Matter 2, 5317 (1990).

${ }^{33}$ J. R. Smith, G. Bozzolo, A. Banerjea, and J. Ferrante, Phys. Rev. Lett. 63, 1269 (1989).

${ }^{34}$ U. Landman, W. D. Luedtke, N. A. Burnham, and R. J. Colton, Science 248, 454 (1990); J. Vac. Sci. Technol. B 9, 414 (1991).

${ }^{35}$ H. J. Kreuzer, Surf. Sci. 246, 336 (1991).

${ }^{36}$ I. W. Lyo and P. Avouris, Science 245, 1369 (1989); 253, 173 (1991).

${ }^{37}$ H. J. Mamin, P. H. Guethner, and D. Rugar, Phys. Rev. Lett. 65, 2418 (1990).

${ }^{38}$ D. M. Eigler and E. K. Schweizer, Nature 344, 524 (1990); D. M. Eigler, C. P. Lutz, and W. E. Rudge, ibid. 352, 600 (1991); J. A. Stroscio and D. M. Eigler, Science 254, 1399 (1991).

${ }^{39}$ J. K. Gimzewski and R. Möller, Phys. Rev. B 36, 1284 (1987).

${ }^{40} \mathrm{~S}$. Ciraci and E. Tekman, Phys. Rev. B 40, 11969 (1989); E. Tekman and S. Ciraci, ibid. 43, 7145 (1991).

${ }^{41} \mathrm{~S}$. Ciraci, Ultramicroscopy 42-44, 16 (1992); E. Tekman and S. Ciraci, Phys. Rev. B 42, 1860 (1990).

${ }^{42}$ N. Garcia (unpublished); see N. Garcia and L. Escapa, Appl. Phys. Lett. 54, 14918 (1989).

${ }^{43}$ N. D. Lang, Phys. Rev. B 36, 8173 (1987); 37, 10395 (1988).

${ }^{44}$ J. Ferrer, A. Martin-Rodero, and F. Flores, Phys. Rev. B 38, 10113 (1988).

${ }^{45}$ G. Doyen, E. Koetter, J. P. Vigneron, and M. Scheffler, Appl. Phys. A 51, 281 (1990).

${ }^{46}$ E. Tekman and S. Ciraci, Phys. Rev. B 40, 8559 (1989); 42, 3098 (1990). A similar situation was observed experimentally by I. W. Lyo and P. Avouris, Science 245, 1369 (1989).

${ }^{47} \mathrm{~J}$. Wintterlin, J. Wiechers, H. Brune, T. Gritsch, H. Höfer, and R. J. Behm, Phys. Rev. Lett. 62, 59 (1989).

${ }^{48}$ J. Bardeen, Phys. Rev. Lett. 6, 57 (1961).

${ }^{49}$ J. Tersoff and D. R. Hamann, Phys. Rev. Lett. 50, 1998 (1983); Phys. Rev. B 31, 805 (1985).

${ }^{50}$ C. J. Chen, J. Phys. Condens. Matter 3, 1227 (1991).

${ }^{51}$ N. D. Lang, Phys. Rev. Lett. 56, 1165 (1986).

${ }^{52}$ F. Flores, A. M. Rodero, E. C. Goldberg, and J. C. Duran, Nuovo Cimento 10, 303 (1988).

${ }^{53}$ J. Harris, Phys. Rev. B 31, 1770 (1985); E. Zaremba and W. Kohn, ibid. 15, 1769 (1977).

${ }^{54}$ J. R. Smith, Phys. Rev. B 181, 522 (1963); J. Ferrante and J. R. Smith, Surf. Sci. 38, 77 (1973).

${ }^{55} \mathrm{P}$. Hohenberg and W. Kohn, Phys. Rev. B 136, B864 (1964).

${ }^{56}$ J. Ferrante and J. R. Smith, Phys. Rev. B 19, 3911 (1979).

${ }^{57}$ See, for instance, E. O. Kane, in Tunneling Phenomena in Solids, edited by E. Burstein and S. Lundqvist (Plenum, New York, 1969).

${ }^{58}$ E. Tekman, Phys. Rev. B 46, 4938 (1992). 